\title{
How Adequate is the Adequate Kidney Function for Clinical Trial Enrollment of Novel Anticancer Drugs?
}

\author{
Nehal Gamal ${ }^{1}$, Kyrillus S. Shohdy ${ }^{2}$ \\ ${ }^{1}$ Medical Student, Kasr Al-Ainy School of Medicine, Cairo University, Cairo, Egypt; ${ }^{2}$ Hematology \\ and Medical Oncology Division, Department of Medicine, Weill Cornell Medical College, New York, \\ $N Y, U S A$
}

\begin{abstract}
Excessively restrictive eligibility criteria hinder the enrollment of cancer patients to clinical trials. Those patients are supposed to represent the range of characteristics of the overall population with particular cancer. This, in turn, affects the generalizability of results and efficiency of the drug development process in oncology. Recent guidelines from the American Society of Clinical Oncology recommend broadening the eligibility criteria for early and late phase trials. A clearly defined rationale is essential for excluding patients. They selected specific items that commonly lead to the exclusion of patients from clinical trials, namely brain metastases, the minimum age for enrollment, human immunodeficiency virus infection, prior and concurrent malignancies, and organ dysfunction. We reviewed the clinical trials of abemaciclib and tucatinib, two novel anticancer agents, to investigate the eligibility criteria used in their ongoing trials and how the cut-offs for adequate kidney functions were defined. We provided recommendations to allow for enrollment of otherwise eligible patients.
\end{abstract}

Keywords: Abemaciclib, Clinical trials, Eligibility criteria, Kidney function, Tucatinib

Corresponding Author: Kyrillus Shohdy, MD; Weill Cornell Medicine, 1300 York Ave, NY, New York, USA; Email: kss4001@med.cornell.edu

Submitted: 19-June-2020, Revised: 17-July-2020, Accepted: 18-July-2020, Published online: 21-July-2020

(cc) BY

\section{Introduction}

Most of the trials of the novel cancer therapeutics have excluded patients with moderate to severe renal dysfunction based on the serum creatinine (SCr) level. There is no standard cutoff for adequate kidney function. Creatinine clearance $(\mathrm{CrCl})$ provides a more accurate assessment ${ }^{1}$. However, SCr within 1.5 times the upper limit of normal is often required for a patient to be eligible for a clinical trial ${ }^{2}, 3$. Clinical practice guidelines for evaluation of kidney function and drug dosing now emphasize using estimated glomerular filtration rate (eGFR) rather than estimated creatinine clearance $(\mathrm{eCrCl})$ or $\mathrm{SCr}{ }^{4,5}$. Using $\mathrm{SCr}$ and eGFR or $\mathrm{eCrCl}$ can be logically inconsistent as a patient with an eligible $\mathrm{SCr}$ may be ineligible by eGFR or $\mathrm{eCrCl}^{6}$. The variability in the definition of kidney function threshold is preventing a significant proportion of otherwise eligible patients from enrolling in clinical trials.
Abemaciclib is an inhibitor of cyclin-dependent kinases 4 and 6 that is recently approved for metastatic breast cancer treatment ${ }^{2}$. Clinical studies showed that abemaciclib causes reversible increases in $\mathrm{SCr}$ due to inhibition of renal tubular secretion transporters, without affecting glomerular function. Thus, for eligibility in clinical trials, alternative markers such as blood urea nitrogen (BUN), cystatin $\mathrm{C}$, or measured glomerular filtration rate (mGFR) using radioisotope studies, which are not dependent on creatinine, should be considered ${ }^{8,9}$.

Tucatinib, a kinase inhibitor used in HER2+ breast cancer, may cause a transient increase in serum creatinine ${ }^{10}$.

Determining Adequate Kidney Function in Abemaciclib and Tucatinib Clinical Trials 
We have queried clinicaltrials.gov on abemaciclib and retrieved 81 clinical trials recruiting 15,336 patients. All except one conducted in the adult population. Thirty-three (43\%) were phase I or phase I/II trials. The median number of enrolled patients was 78 (range: 6-4580). Most (95\%) of the studies were interventional. Abemaciclib was tested as monotherapy in $34(42 \%)$ of the studies.

Thirty-seven (46\%) studies reported the exact method and cutoff value for determining adequate kidney function. These methods included $\mathrm{SCr}, \mathrm{CrCl}$ estimated by Cockcroft-Gault, glomerular filtration rate (GFR) estimated by The Modification of Diet in Renal Disease Study (MDRD) equation, $\mathrm{CrCl}$ using 24hour urine collection, or $\mathrm{CrCl}$ calculated using method standard for the institution. Twenty (25\%) studies restricted their eligibility to only one method, and in 12 studies, the method was SCr. Sixteen (20\%) studies adopted two methods. Only one trial included three different methods for eligibility, which are estimated $\mathrm{CrCl} \geq 30 \mathrm{~mL} / \mathrm{min}$ by Cockcroft-Gault equation or eGFR $\geq 30 \mathrm{~mL} / \mathrm{min} / 1.73$ $\mathrm{m}^{2}$ by MDRD or Chronic Kidney Disease Epidemiology Collaboration (CKD-EPI) formula, or actual $\mathrm{CrCl}$ as determined by 24-hour urine collection.

Our analysis showed that approximately half of the studies did not report the exact method and cutoffs for determining adequate kidney function in their published protocol on clinicaltrials.gov. The studies were usually restricted to one method and less likely to use multiple methods.

We found much heterogeneity and imprecision in the definition of kidney function. Based on our analysis, only $46 \%$ of studies reported the exact method and cutoff values for determining adequate kidney function. Twelve (32\%) of them depended on $\mathrm{SCr}$ alone, while the American Society of Clinical Oncology (ASCO) guidelines recommend using $\mathrm{CrCl}$ rather than $\mathrm{SCr}$ levels ${ }^{1}$.

Interestingly, only one trial of 81 was flexible to include three different methods for estimation. Although using more than one method can increase largely the probability of a given patient to meet the eligibility criteria, the feasibility of application remains a challenge, especially in limited-resource settings and investigator-initiated trials.

Abemaciclib has measurements to cause reversible and consistent increases in SCr levels of around $15-40 \%$ over baseline in the studied subjects ${ }^{8}$. Chappell et al ${ }^{9}$ conducted a randomized, placebocontrolled crossover study on abemaciclib in healthy subjects in addition to in-vitro analysis of transporter interaction and inhibitory studies. They concluded that abemaciclib inhibits renal tubular secretion without changing the glomerular filtration rate. The process is mediated through inhibition of organic cation transporter 2, multidrug and toxin extrusion (MATE) 1, and MATE2-K transporters that mediate active secretion of creatinine from the proximal tubule. Thus, abemaciclib does not cause damage to nephron or directly affect kidney function. Therefore, cystatin $\mathrm{C}$ or other measures of glomerular filtration rate, which are independent of proximal tubule secretory transporters, are needed to evaluate the kidney function for patients receiving abemaciclib ${ }^{9}$. Taking all of that into consideration, most of the clinical trials have not provided enough experimental measures to determine precise cutoff levels for eligibility of patients with kidney function impairment.

Murthy et al ${ }^{10}$ reported a similar condition with tucatinib, another kinase inhibitor that is being developed as a novel HER2+ breast cancer treatment. Tucatinib was showed to increase metformin plasma exposure by $48 \%$ and to cause a transient increase in serum creatinine level without any effect on kidney function. This elevation in serum creatinine is due to inhibition of tubular secretion of creatinine via OCT2 and MATE1, which is similar to the abemaciclib effect ${ }^{10}$. Clinical trials on tucatinib also showed the same heterogeneity in their eligibility criteria for kidney function as abemaciclib. Only 4 out of the 15 clinical trials registered in clinicaltrials.gov, reported an exact cutoff value for kidney function, and they used different measures and values.

\section{Which Method to Assess Renal Function Adequacy?}

Patients with renal impairment are often excluded from phase I trials without enough understanding of drug pharmacokinetics and the actual possibility to cause direct harm to kidneys. However, protocol amendments are rarely considered when this data becomes available after progress in drug development ${ }^{11,12}$.

In clinical trials, $\mathrm{SCr}$ may not accurately reflect kidney function, so calculated $\mathrm{CrCl}$ of $<60 \mathrm{~mL} / \mathrm{min}$ should be required to be eligible. Although measured $\mathrm{CrCl}$ is more accurate, it's often impractical due to difficulties related to the urine 
collection process. The cost of the radionuclide assessment of $\mathrm{CrCl}$ represents another challenge ${ }^{11}$. The Cockcroft-Gault and the MDRD equations are the most frequently used formulae to estimate $\mathrm{CrCl}$. Although the MDRD equation is recommended over the Cockcroft-Gault equation, according to the National Kidney Foundation, the clinical difference is insignificant when compared with GFR and with consideration to pharmacokinetics ${ }^{13}$.

Due to the nature of research in oncology, a large proportion of populations are from older age groups. A significant issue with aging arises from the physiological decline in kidney function, which may be exacerbated by their disease conditions. Those patients are susceptible to exclusion from trials without evaluating the drug safety and efficacy on them. However, that same drug may be prescribed to them later on ${ }^{14}$.

A retrospective analysis of the Gynecologic Oncology Group 182 trial concluded that a cutoff value of $\mathrm{CrCl}>60 \mathrm{~mL} / \mathrm{min}$ would exclude $15 \%$ of patients from treatment ${ }^{15}$. On the other hand, The National Cancer Institute concludes that patients with mild kidney dysfunction can be enrolled without a significant increase in the risk of toxicity 16. This creates a conflict between the current practices and what should be done and makes us question the current eligibility policies and seek more precise guidelines.

It is very important to provide a clear definition of what "adequate kidney function" means with exact cutoff value to avoid unnecessary exclusion of eligible patients and to guarantee patient safety. The ASCO's call for modernizing clinical trial eligibility criteria recommends that patients with lower $\mathrm{CrCl}$ values, less than $30 \mathrm{~mL} / \mathrm{min}$, should be included in trials as long as renal toxicity and clearance are not directly related to the treatment ${ }^{17}$. They also recommend $\mathrm{CrCl}$ assessment, using The CockcroftGault and MDRD equations, over SCr. It's necessary to apply consistent measurements throughout the development process of the drug.

\section{Conclusion}

In conclusion, additional studies are essential to investigate the validity of the currently used measures of kidney function in general and for abemaciclib in particular. Most of the eligibility criteria used in the ongoing clinical trials are not based on strong evidence with largely arbitrary cut- offs. This deprives many cancer patients to enroll in those trials. Until eligibility guidelines of more accurate estimates are developed, using more than one estimate should be adopted in clinical trials of novel cancer therapeutics.

\section{Acknowledgment}

We would like to acknowledge George W. Sledge Jr., M.D. (Stanford University Medical Center), and Bishoy Faltas, M.D. (Weill Cornell Medicine) for their insightful discussions.

\section{Authors' contribution}

Conceived and designed the analysis (NG and KSS); Collected the data (NG); Wrote the manuscript (NG); Approved the final version of the manuscript (NG and KSS).

\section{Conflict of interest}

The authors declare that there is no conflict of interest.

\section{Data availability}

None.

\section{Funding}

KS was funded by a Conquer Cancer Foundation of ASCO Long-term International Fellowship, supported by the Mission Endowment of ASCO's Conquer Cancer Foundation. Any opinions, findings, and conclusions expressed in this material are those of the author(s) and do not necessarily reflect those of the American Society of Clinical Oncology ${ }^{\circledR}$ or Conquer Cancer.

\section{Study registration}

None.

\section{References}

1. Kim ES, Bruinooge SS, Roberts S, et al. Broadening eligibility criteria to make clinical trials more representative: American Society of Clinical Oncology and Friends of Cancer Research joint research statement. J Clin Oncol. 2017; 35(33): 3737-3744.

2. Malik L, Lu D. Eligibility criteria for phase I clinical trials: tight vs loose? Cancer Chemother Pharmacol. 2019; 83(5): 999-1002.

3. Perrone RD, Madias NE, Levey AS. Serum creatinine as an index of renal function: new insights into old concepts. Clin Chem. 1992; 38(10): 1933-1953.

4. U.S. Food and Drug Administration. Guidance Document: Population Pharmacokinetics. 2019. https://www.fda.gov/regulatory-information/searchfda-guidance-documents/populationpharmacokinetics. Last access date: 20-July-2020.

5. Kidney Disease: Improving Global Outcomes (KDIGO) CKD-MBD Update Work Group. KDIGO 2017 clinical practice guideline update for the diagnosis, evaluation, prevention, and treatment of chronic kidney diseasemineral and bone disorder (CKD-MBD). Kidney Int Suppl. 2017; 7(1): 1-59.

6. Wang E, Paulus JK, Hackenyos D, Inker LA, Levey AS, Mathew P. Imprecise kidney function thresholds in cancer clinical trials and the potential for harm. JNCI 
Cancer Spectr. 2018; 2(4): pky060.

7. Johnston S, Martin M, Di Leo A, et al. MONARCH 3 final PFS: a randomized study of abemaciclib as initial therapy for advanced breast cancer. NPJ Breast Cancer. 2019; 5: 5.

8. Eli Lilly/ Summary of product characteristics (Verzenio)/. $2017 . \quad$ p. $1-23$. http://pi.lilly.com/ca/verzenio-ca-pm.pdf. Last access date: 20-July-2020.

9. Chappell JC, Turner PK, Pak YA, et al. Abemaciclib inhibits renal tubular secretion without changing glomerular filtration rate. Clin Pharmacol Ther. 2019; 105(5): 1187-1195.

10. Murthy RK, Loi S, Okines A, Paplomata E, et al. Tucatinib, trastuzumab, and capecitabine for her2positive metastatic breast cancer. N Engl J Med. 2020; 382(7): 597-609.

11. Launay-Vacher V, Oudard S, Janus N, et al. Prevalence of renal insufficiency in cancer patients and implications for anticancer drug management: The renal insufficiency and anticancer medications (IRMA) study. Cancer. 2007; 110(6): 1376-1384.

12. Bluethmann SM, Mariotto AB, Rowland JH. Anticipating the "silver tsunami": Prevalence trajectories and comorbidity burden among older cancer survivors in the United States. Cancer Epidemiol Biomarkers Prev. 2016; 25(7): 1029-1036.

13. Coresh J, Stevens LA. Kidney function estimating equations: Where do we stand? Curr Opin Nephrol Hypertens. 2006; 15(3): 276-284.

14. Hurria A, Levit LA, Dale W, et al. Improving the evidence base for treating older adults with cancer: American Society of Clinical Oncology statement. J Clin Oncol. 2015; 33(32): 3826-3833.

15. Bookman MA, Brady MF, McGuire WP, et al. Evaluation of new platinum-based treatment regimens in advanced-stage ovarian cancer: A phase III trial of the Gynecologic Cancer Intergroup. J Clin Oncol. 2009; 27(9): 1419-1425.

16. Beumer JH, Ding F, Tawbi H, et al. Effect of renal dysfunction on toxicity in three decades of cancer therapy evaluation program-sponsored single-agent phase I studies. J Clin Oncol. 2016; 34(2): 110-116.

17. Lichtman SM, Harvey RD, Damiette Smit MA, et al. Modernizing clinical trial eligibility criteria: Recommendations of the American Society of Clinical Oncology - Friends of Cancer Research organ dysfunction, prior or concurrent malignancy, and comorbidities working group. J Clin Oncol. 2017; 35(33): 3753-3759. 